\title{
To Random Access Or Schedule? Optimum 3GPP Licensed-Assisted Access for Machine-to-Machine Communications
}

\author{
Shao-Yu Lien* and Yueh Jir Wang** \\ *Department of Electronic Engineering, National Formosa University, Yulin 63201, Taiwan, Email: sylien@nfu.edu.tw \\ **Information and Communication Research Division, National Chung-Shan Institute of Science and Technology, \\ Taoyuan, Taiwan, Email: easonwang0218@yahoo.com.tw
}

\begin{abstract}
To support various Internet-of-Thing (IoT) applications involving an enormous number of machine-type devices, deploying LTE/LTE-A networks on ISM bands with an extensive bandwidth has been regarded as a promising solution to enable machine-to-machine (M2M) communications. Designating as licensed-assisted access (LAA) in 3GPP Rel-13, unprecedented challenge of uncontrollable interference from collocated WiFi networks, however, obstructs the state-of-the-art scheduling based radio resource access in LTE/LTE-A. To practice LAA, in this paper, we shall derive the optimum radio access scheme for the urgent needs of 3GPP Rel-13 standardization. Observing the LAA-WiFi hidden terminal problem, we analytically derive the optimum number of LAA users randomly accessing a pool of radio resources and the optimum condition for random access, to reach a superior throughput of a LAA network as compared with that of a scheduling based scheme. Our analytic results demonstrate that the conventional scheduling based scheme in LTE/LTE-A may not always be optimal, and thus our proposition leads to the optimum radio access design for LAA networks.

Index Terms-M2M communications, LTE/LTE-A, LAA, IoT applications.
\end{abstract}

\section{INTRODUCTION}

An urgent issue which will be suffered by the state-of-theart cellular networks in 2020 lies in the emergence of billions of heterogeneous devices. In addition to conventional user equipments (UEs) supporting human-to-human communications, an immense number of low-cost devices for machine-tomachine (M2M) communications will be further introduced to support a variety of Internet-of-Thing (IoT) applications. As a consequence, the traffic volume is projected to increase a thousand-fold in 2020.

To tackle such unprecedented challenge of supporting an immense amount of IoT traffic, two categories of schemes are currently studied. The first category is to extensively investigate the traffic characteristics of M2M communications [1], [2]. These studies reveal that IoT applications (such as sensors, meters, or actuators) may only generate few coded bits of data. By using the state-of-the-art mechanisms in UMTS and LTE/LTE-A requiring heavy signaling overheads, it may result in low spectrum efficiency to support the transmissions of small size data. To enhance the spectrum efficiency, reducing signaling overheads in the air interface has been one of the major design goals of machine-type communications in 3GPP Release 13. In addition to alleviate signaling overheads, novel technologies, such as non-orthogonal multiple access (NOMA) [3], multiuser superposition transmission (MUST) [4], and full-duplex radios [5], are widely discussed to further boost spectrum efficiency. However, even though with those facilitating technologies, to support an immense amount of IoT traffic, communication bandwidth still shall be largely extended. This requirement drives the second category of schemes to utilize unlicensed ISM bands.

Since 2015, 3GPP has launched the standardization progress of deploying LTE-A networks on the $5 \mathrm{GHz}$ unlicensed bands, which is known as the License-Assisted Access (LAA) technology in Release 13. Although LTE-A networks may enjoy a wide bandwidth via exploiting unlicensed bands, there are three obstacles unfavorable for the mobile network deployment. (i) Transmission behaviors on the unlicensed bands shall adhere communication regulations imposed by different countries. These communication regulations include the maximum transmission power limit, power spectral density limit, and/or out-of-band emission limit. These regulations significantly limit the communication range of mobile networks. Therefore, LAA is only feasible for small cell deployments such as femtocells or picocells. (ii) There are two mayor interference sources on the $5 \mathrm{GHz}$ unlicensed bands: WiFi networks (IEEE 802.11a, IEEE 802.11ac, IEEE 802.11ax) and weather radar systems. As a result, communications on the unlicensed bands may be unreliable, which is particularly harmful for control signaling exchanges in LAA. To tackle this issue, carrier aggregation (CA) has been adopted as a mandatory technology for LAA. Specifically, radio resource control (RRC) signaling is performed via the primary carrier on the licensed bands, while data exchanges are performed via the secondary carrier on the unlicensed bands. (iii) According to the communication regulation in Japan and Europe, for transmissions on the unlicensed bands, any transmitter shall perform clear channel assessment (CCA) before transmissions. Transmissions are able to be performed only if the channel is sensed to be idle. However, this mandatory procedure may invoke a particular hidden terminal problem between a WiFi network and a LAA network. Such a hidden terminal problem consequently motivates us to investigate the performance of LAA coexisting with WiFi networks.

It is well known that LTE-A networks adopt a schedul- 


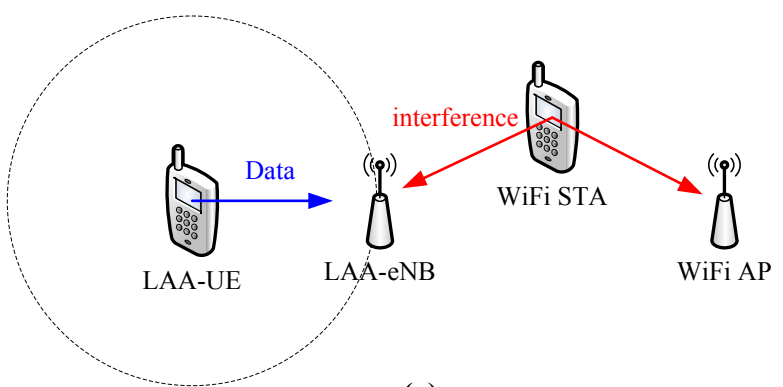

(a)

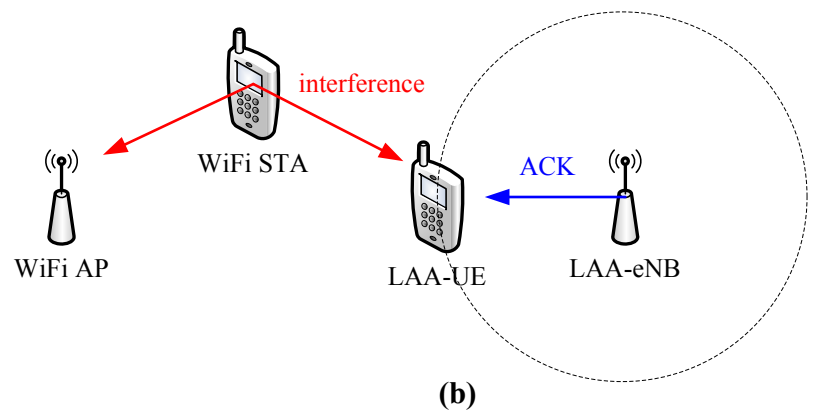

Fig. 1. (a) Before transmitting data, a LAA transmitter (taking a LAA-UE as a elaboration example) needs to perform CCA. However, even though a clear channel is sensed, there may be interference at the LAA receiver. (b) Even though a LAA receiver successfully receives data, it needs to reply ACK. However, this ACK may not be received by a LAA transmitter due to $\mathrm{WiFi}$ interference.

ing based radio resource access, while WiFi networks adopt the carrier sense multiple access with collision avoidance (CSMA/CA) based random access. The presence of the LAAWiFi hidden terminal problem raises a fundamental question whether a scheduling based radio resource access is optimum for a LAA network to coexist with WiFi networks. In this paper, we consequently investigate the performance of a LAA network coexisting with WiFi networks, and derive the optimum radio access scheme for $\mathrm{M} 2 \mathrm{M}$ communications using LAA. Our result paves the foundation for the radio access design of LAA networks.

\section{LAA-WIFI Hidden TERMINAL PRoblem AND SYSTEM CONSIDERATIONS}

\section{A. LAA-WiFi Hidden Terminal Problem}

According to the communication regulations in Japan and Europe, a LAA transmitter shall perform CCA. If the channel is sensed to be busy, then it suggests that there should be a WiFi transmitter currently transmitting data. It may also suggest that there is a WiFi receiver currently receiving data. To avoid interference to/from a WiFi network, the LAA transmitter is not able to transmit data. Transmissions from a LAA transmitter can take place only if the channel is sensed to be idle.

- Interference levels suffered by a LAA transmitter and a LAA receiver may be largely different. Since a LAA transmitter and a LAA receiver may be geographically separated apart, a clear channel sensed at the LAA transmitter side does not suggest that the channel is also clear at the LAA receiver side, as shown in Fig. 1(a). Even though the data transmission can be successfully received by a LAA receiver, it shall report an acknowledgement (ACK) to the LAA transmitter. It does not suggest that this ACK is able to be successfully received by the LAA transmitter, as shown in Fig. 1(b). This phenomenon is known as the LAA-WiFi hidden terminal problem.

- Multiple Access with Collision Avoidance (MACA) may not be feasible for $\mathrm{LAA}$-WiFi coexistence. In WiFi networks, request-to-send (RTS) and clear-to-send (CTS) messages are exploited to avoid the hidden terminal problem. However, since there is no air interface for information exchanges between a WiFi network and a LAA network, this RTS-CTS exchange scheme known as MACA may not be utilized by a LAA network to avoid the LAA-WiFi hidden terminal problem.

- Scheduling based radio resource access may not be reliable for LAA data communications. For uplink transmissions in a LAA network composes of a LAAeNB and multiple LAA-UEs, the LAA-eNB allocates a specific amount of radio resources for each LAA-UE. However, when the LAA-eNB announces the resource allocation for all LAA-UEs, it does not know whether a radio resource allocating to the $k$ th LAA-UE is able to be utilized by this $k$ th LAA-UE. Since the $k$ th LAAUE needs to perform CCA before utilizing the allocated radio resource, if this radio resource is occupied by a WiFi network, then transmissions on this radio resource is forbidden. A similar challenge also occurs at the downlink transmissions in a LAA network. As a result, the transmission latency in a LAA network may be unacceptable when the conventional scheduling based radio resource access is adopted.

\section{B. Preliminary of the Optimum LAA Radio Resource Access Design}

Since LAA adopts the orthogonal frequency division multiple access (OFDMA), radio resources are allocated in the basic unit of a resource block (RB). As each transmission of an IoT application may only carry a few coded bits, each LAAUE may only need a few RBs for each uplink transmission. These RBs used for one uplink transmission is referred as one resource batch. In this paper, uplink transmissions in a LAA network composed of a LAA-eNB and $N$ LAAUEs are considered. According to state-of-the-art scheduling based radio resource access adopted by LTE-A, if a LAA-UE requests for one resource batch, the LAA-eNB will allocate exactly one resource batch for the LAA-UE.

Definition 1. (Throughput of a LAA-UE) For a LAA-UE needing one radio batch for uplink transmissions, the throughput of the LAA-UE is defined as the expected number of resource batches used by this $L A A-U E$ without interference.

Due to the mobile and random geographic locations of $\mathrm{WiFi}$ networks and LAA-UEs, interference from WiFi networks to a LAA network is stochastic. Consider that the probability 


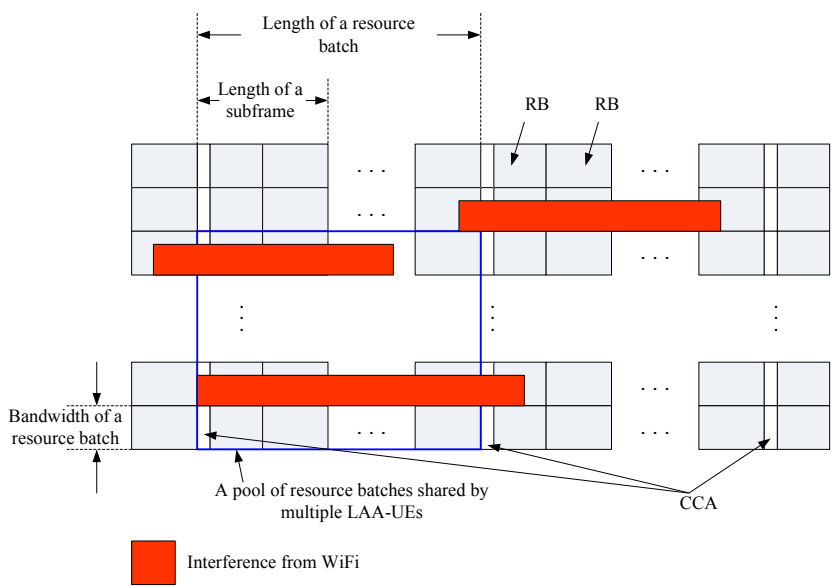

Fig. 2. For LAA, if a resource batch is occupied by WiFi networks, it can not be utilized by a LAA-UE. A LAA-eNB may allocate multiple resource batches in the frequency domain to LAA-UE(s) to avoid interference from WiFi.

of interference occurrence from WiFi networks at each radio resource is independently and identically distributed (i.i.d.) $p$. If a LAA-UE requests for one resource batch and the LAAeNB allocates exactly one resource batch for this LAA-UE, the throughput of this LAA-UE is $1-p$, which completely depends on the probability that this resource batch is occupied by WiFi networks.

To enhance the throughput and thus the latency performance of a LAA-UE, a promising scheme is to exploit diversity. Consider that there are total $K$ resource batches available in the frequency domain, indexed by $k=1, \ldots, K$, for the LAA-eNB to be allocated to LAA-UEs, as shown in Fig. 2. If a LAA-UE needs one resource batch while the LAA-eNB allocates two resource batches for this LAA-UE, then this LAA-UE is able to successfully transmit data to the LAAeNB if both resource batches are not simultaneously occupied by WiFi networks. As a result, the throughput of the LAA$\mathrm{UE}$ is $1-p^{2}$. As $1-p^{2} \geq 1-p$, the LAA-UE throughput of this scheme can be larger than the conventional scheduling based radio resource access scheme. If the LAA-eNB allocates three resource batches for a LAA-UE needing one resource batch, then the throughput of the LAA-UE is $1-p^{3}$, which is further enhanced. Although the diversity scheme is able to significantly enhance the throughput of a LAA-UE, the network throughput may be severely degraded.

Definition 2. (Throughput of a LAA network) The throughput of a LAA network is defined by

$$
\rho=\mathbb{E}\left[K^{\prime}\right],
$$

where $K^{\prime}$ is number of resource batches without interference (not occupied by WiFi networks). $\mathbb{E}\left[K^{\prime}\right]$ is the expected value of $K^{\prime}$.

If the LAA-eNB allocates one resource batches for a LAAUE needing one resource batch, then the throughput of the LAA network is $1-p$. On the other hands, if the LAA-eNB exploits diversity to allocate two resource batches for a LAAUE needing one resource batch, then the throughput of the LAA network is $\frac{1-p^{2}}{2}$, which may be lower than $1-p$.

To further enhance the throughput of a LAA network, denote $\mathcal{K}$ as a set of resource batches allocating to the $i$ th LAA-UE and $\|\mathcal{K}\|=M$ is the cardinality of $\mathcal{K}$ (where $M \leq K)$, the LAA network may allocate these resource batches $\mathcal{K}$ to other LAA-UEs. In this case, $\mathcal{K}$ form a resource pool, which can be utilized by multiple LAA-UEs. Among this resource pool, each LAA-UE performs CCA at all $m$ resource batches, and selects one resource batch sensed to be idle to transmit data. If all LAA-UEs utilize different resource batches to transmit data, then these resource batches $\mathcal{K}$ can be fully utilized to enhance the throughput of the LAA network. However, if some of LAA-UEs unfortunately select the same unoccupied resource batch to transmit data, then collisions occur and the throughput of LAA-UEs may be degraded. Consequently, there is a tradeoff between the throughput of the LAA network and the throughput of each individual LAA-UE.

The engineering issue of maximizing the throughput of a LAA-UE is not difficult, as it is proportional to the number of resource bathes specifically allocating to a LAA-UE. However, maximizing the throughput of a LAA network is a critical challenge. In the following section, we emphasize on finding the optimum number of LAA-UEs to share a resource pool such that the throughput of the LAA network is maximized.

\section{Optimum LAA RADIO RESOURCE ACCESS}

Given a resource pool $\mathcal{K}$ composed of $M$ resource batches indexed by $m=1, \ldots, M$, these $M$ resource batches are shared by $N$ LAA-UEs indexed by $i=1 \ldots, N$. Before transmitting data, each LAA-UE shall perform CCA on all $M$ resource batches, and select one unoccupied resource batch to transmit data. Suppose that the probability of interference occurrence from WiFi networks at each resource batch for all LAA-UEs is i.i.d. (denoted as $p$ ). Let

$$
\mathbf{I}_{m, i}=\left\{\begin{array}{l}
1, i \text { th LAA-UE selects the } m \text { th resource batch, } \\
0, \text { otherwise }
\end{array}\right.
$$

be an indication function. The throughput of a LAA network can be obtained by the following theorem.

Theorem 1. The throughput of a LAA network is given by

$$
\rho=M N\left(\frac{1-p^{M}}{M}\right)\left(1-\frac{1-p^{M}}{M}\right)^{N-1} .
$$

Proof: Since the probability that the $i$ th LAA-UE has one unoccupied (no interference from WiFi networks) resource batch to transmit data is given by

$$
\operatorname{Pr}\left\{\sum_{m=1}^{M} \mathbf{I}_{m, i}=1\right\}=1-p^{M},
$$

while the probability that the $i$ th LAA-UE has no (no interference from WiFi networks) resource batch to transmit data 
is given by

$$
\operatorname{Pr}\left\{\sum_{m=1}^{M} \mathbf{I}_{m, i}=0\right\}=p^{M},
$$

we obtain the expected value of $\sum_{m=1}^{M} \mathbf{I}_{m, i}$ as

$$
\mathbb{E}\left[\sum_{m=1}^{M} \mathbf{I}_{m, i}\right]=\sum_{m=1}^{M} \mathbb{E}\left[\mathbf{I}_{m, i}\right]=M \mathbb{E}\left[\mathbf{I}_{m, i}\right]=1-p^{M} .
$$

Thus, we have

$$
\mathbb{E}\left[\mathbf{I}_{m, i}\right]=\operatorname{Pr}\left\{\mathbf{I}_{m, i}\right\}=\frac{1-p^{M}}{M} .
$$

For each LAA-UE, the probability that the $m$ th resource batch is selected (suffering no interference from WiFi networks) and no collision occurs at this resource batch (no interference from other LAA-UEs) is given by

$$
\operatorname{Pr}\left\{\sum_{i=1}^{N} \mathbf{I}_{m, i}=1\right\}=N\left(\frac{1-p^{M}}{M}\right)\left(1-\frac{1-p^{M}}{M}\right)^{N-1},
$$

which is the throughput of the $m$ th resource batch. The throughput of a LAA network allocating $M$ resource batches is therefore given by (3).

Given a resource pool composed of $M$ resource batches, the optimum number of LAA-UEs sharing these resource batches is obtained by solving the following optimization

$$
N^{*}=\arg \max _{N}\left\{M N\left(\frac{1-p^{M}}{M}\right)\left(1-\frac{1-p^{M}}{M}\right)^{N-1}\right\} .
$$

Theorem 2. The optimum number of LAA-UEs sharing $M$ resource batches is

$$
N^{*}=\left\{\begin{array}{l}
\frac{M}{1-p^{M}}, \text { if } \frac{M}{1-p^{M}} \text { is an integer } \\
\left\lfloor\frac{M}{1-p^{M}}\right\rfloor, \text { otherwise. }
\end{array}\right.
$$

Proof: Let the throughput of a LAA network $\rho$ be a function of $N, \rho=f(N)$, the optimum $N^{*}$ satisfies the following two conditions. First,

$$
\begin{aligned}
f\left(N^{*}\right) & -f\left(N^{*}-1\right) \\
& =M N^{*}\left(\frac{1-p^{M}}{M}\right)\left(1-\frac{1-p^{M}}{M}\right)^{N^{*}-1} \\
& -M\left(N^{*}-1\right)\left(\frac{1-p^{M}}{M}\right)\left(1-\frac{1-p^{M}}{M}\right)^{N^{*}-2} \\
& =M\left[1-N^{*} \frac{1-p^{M}}{M}\right] \frac{1-p^{M}}{M}\left(1-\frac{1-p^{M}}{M}\right)^{N^{*}-2} \\
& \geq 0,
\end{aligned}
$$

and we therefore obtain

$$
\left[1-N^{*} \frac{1-p^{M}}{M}\right] \geq 0 \Leftrightarrow N^{*} \leq \frac{M}{1-p^{M}} \Rightarrow N^{*} \leq\left\lfloor\frac{M}{1-p^{M}}\right\rfloor
$$

for $N^{*} \in \mathbb{Z}^{+}$. Second,

$$
\begin{aligned}
f\left(N^{*}\right) & -f\left(N^{*}+1\right) \\
& =M N^{*}\left(\frac{1-p^{M}}{M}\right)\left(1-\frac{1-p^{M}}{M}\right)^{N^{*}-1} \\
& -M\left(N^{*}+1\right)\left(\frac{1-p^{M}}{M}\right)\left(1-\frac{1-p^{M}}{M}\right)^{N^{*}} \\
& =M\left[\left(N^{*}+1\right) \frac{1-p^{M}}{M}-1\right] \frac{1-p^{M}}{M}\left(1-\frac{1-p^{M}}{M}\right)^{N^{*}-1} \\
& \geq 0,
\end{aligned}
$$

then we have

$$
\begin{gathered}
{\left[\left(N^{*}+1\right) \frac{1-p^{M}}{M}-1\right] \geq 0 \Leftrightarrow N^{*} \geq \frac{1-\frac{1-p^{M}}{M}}{\frac{1-p^{M}}{M}}} \\
\Rightarrow N^{*} \geq\left\lceil\frac{M}{1-p^{M}}\right\rceil-1 .
\end{gathered}
$$

Since

$$
\left\lfloor\frac{M}{1-p^{M}}\right\rfloor \geq N^{*} \geq\left\lceil\frac{M}{1-p^{M}}\right\rceil-1,
$$

(10) can be obtained.

Although a scheduling based radio resource access is conventionally regarded as an effective scheme to enhance the throughput, the performance may degrade when WiFi networks are active. Theorem 2 reveals a significant engineering insight that multiple LAA-UEs sharing a resource pool are able to enhance the throughput of a LAA network. This performance enhancement is boosted by fully utilizing each resource batch to be potentially accessed by multiple LAAUEs.

Corollary 1. For a pool of resource batches shared by multiple LAA-UEs, each resource batch is able to enjoy a higher utilization as compared with that of a scheduling based radio access.

Proof: In a scheduling based radio access, each resource batch is allocated to only one user. Nevertheless, the result in Theorem 2 reveals that if $M=1$, then

$$
N^{*}=\left\lceil\frac{1}{1-p}\right\rceil \geq 1 \text {. }
$$

In other words, if a resource pool only contains one resource batch, to maximize the throughput of a LAA network, this resource batch shall be shared by more than one LAA-UE. (10) further reveals that, for a resource pool composed of $M$ resource batches,

$$
N^{*}=\left\lceil\frac{M}{1-p^{M}}\right\rceil \geq M,
$$

which suggests that more than $M$ LAA-UEs can be introduced to share $M$ resource batches.

In the following theorems, we further provide the optimum throughput of a LAA network and the throughput of each LAA-UE when a pool of $M$ resource batches is allocated to be shared by $N^{*}$ LAA-UEs. 
Theorem 3. The optimum throughput of a LAA network when $M$ resource batches are allocated to be shared by $N^{*} L A A$ UEs is given by

$\rho^{*}=\left\{\begin{array}{l}M\left(1-\frac{1-p^{M}}{M}\right)^{\frac{M}{1-p^{M}}-1}, \text { if } \frac{M}{1-p^{M}} \text { is an integer } \\ M\left\lfloor\frac{M}{1-p^{M}}\right\rfloor\left(\frac{1-p^{M}}{M}\right)\left(1-\frac{1-p^{M}}{M}\right)^{\left\lfloor\frac{M}{1-p^{M}}\right\rfloor-1}, \text { otherwise. }\end{array}\right.$

Proof: (18) can be obtained by substituting (10) into (3).

Theorem 4. The throughput of each LAA-UE when $M$ resource batches are allocated to be shared by $N^{*} L A A-U E s$ is given by

$$
\eta=\frac{\rho^{*}}{N} .
$$

Proof: Let $Y_{i}$ be the number of resource batches utilized by the $i$ th LAA-UE without interference from WiFi networks and other LAA-UEs, where $Y_{i} \in[0,1]$,

$$
\mathbb{E}\left[\sum_{i=1}^{N} Y_{i}\right]=\rho^{*} \text {. }
$$

We thus obtain

$$
\sum_{i=1}^{N} \mathbb{E}\left[Y_{i}\right]=\rho^{*} \Rightarrow \mathbb{E}\left[Y_{i}\right]=\eta=\frac{\rho^{*}}{N}
$$

In Corollary 1, it suggests that a pool of $M$ resource batches shared by $N^{*}$ LAA-UEs $\left(N^{*} \geq M\right)$ is able to enhance the throughput of a LAA network adopting a scheduling based radio resource access. In the following theorem, the condition of this performance enhancement is provided.

Theorem 5. A pool of $M$ resource batches shared by $N^{*} L A A$ UEs provides a higher throughput of a LAA network than that of a scheduling based radio resource access if

$$
p \geq 1-\left(1-\frac{1}{M}\right)^{M-1} .
$$

Proof: Suppose that $M$ resource batches are utilized by a LAA network, if a scheduling based radio resource access is adopted, then each resource batch is allocated to one LAA-UE. In this case, the throughput of a LAA network is $M(1-p)$. On the other hand, if these $M$ resource batches are shared by $N^{*}$ LAA-UEs, then the optimum throughput of a LAA network can be obtained by (18). Therefore, a random access scheme provides a better throughput performance if

$$
M\left\lfloor\frac{M}{1-p^{M}}\right\rfloor\left(\frac{1-p^{M}}{M}\right)\left(1-\frac{1-p^{M}}{M}\right)^{\left\lfloor\frac{M}{1-p^{M}}\right\rfloor-1} \geq M(1-p)
$$

Since

$$
\begin{aligned}
& M\left\lfloor\frac{M}{1-p^{M}}\right\rfloor\left(\frac{1-p^{M}}{M}\right)\left(1-\frac{1-p^{M}}{M}\right)^{\left\lfloor\frac{M}{1-p^{M}}\right\rfloor-1} \\
& \approx M\left(1-\frac{1-p^{M}}{M}\right)^{\frac{M}{1-p^{M}}-1}
\end{aligned}
$$

we can rewrite (23) as

$$
\begin{aligned}
& M\left(1-\frac{1-p^{M}}{M}\right)^{\frac{M}{1-p^{M}}-1} \geq M(1-p) \\
& \Rightarrow p \geq 1-\left(1-\frac{1-p^{M}}{M}\right)^{\frac{M}{1-p^{M}}-1} \approx 1-\left(1-\frac{1}{M}\right)^{M-1} .
\end{aligned}
$$

We therefore obtain (22).

Based on (22), an optimum strategy of radio access for LAA is obtained by

Proposition 1. A LAA network adopts a conventional scheduling based radio resource access if $p<1-\left(1-\frac{1}{M}\right)^{M-1}$; otherwise, a LAA-eNB allocates a pool of resource batches to be shared by $N^{*}$ LAA-UEs.

\section{Simulation Evaluation}

In this simulation, a LAA network with a $20 \mathrm{MHz}$ bandwidth is considered (i.e., $100 \mathrm{RBs}$ in the frequency domain). As each transmission of IoT applications only carries a few bits, a resource batch is regarded as a RB in the frequency domain. According to the communication regulations on the ISM bands in Japan and Europe, the maximum time for each transmission is $4 \mathrm{~ms}$ (in Japan) or $10 \mathrm{~ms}$ (in Europe). The length of each resource batch is regarded as $4 \mathrm{~ms}$. In this simulation, $120 \mathrm{LAA}-\mathrm{UEs}$ and $50 \mathrm{WiFi}$ stations are randomly and uniformly deployed in an area with an inter-site distance $100 \mathrm{~m}$. The transmission power for a LAA-UE and a WiFi station is both $20 \mathrm{dBm}$. Ay the beginning of each resource batch, the length of CCA is $20 \mu \mathrm{s}$.

In Theorem 2, we first show that the number of LAA-UEs sharing a pool of resource batches composed of $M$ resource batches is $N^{*}$. To show that $N^{*}$ is optimum, in Fig. 3, the throughput of the LAA network under different numbers of LAA-UEs sharing $M=40$ resource batches is provided. It shows that any $N$ deviating from $N^{*}$ results in a lower throughput, since any value of $N$ larger that $N^{*}$ results in over-congestion at each resource batch, while any value of $N$ lower that $N^{*}$ results in under-utilization at each resource batch. These results confirm the optimum $N^{*}$.

In Corollary 1, we next show that, to enhance the throughput of the LAA network, a pool of $M$ resource batches can be shared by $N^{*} \geq M$. To justify this result, in Fig. 4, the optimum numbers of LAA-UEs sharing different numbers of $M$ resource batches are shown. It can be observed that the result in Corollary 1 is fully supported.

Finally, in Theorem 5, we provide the condition that a random access provides a better throughput of a LAA network than that using a scheduling based radio resource access. By exploiting this condition, in Proposition 1, we further derive the optimum LAA as a form of switch between a random access and a conventional scheduling based radio access. To justify these results, in Fig. 5, the simulations results on the throughput of the LAA network using a random access (i.e., a pool of resource batches), a scheduling based radio resource access, and our scheme in Proposition 1 are provided. We can observe from Fig. 5 that, a scheduling 


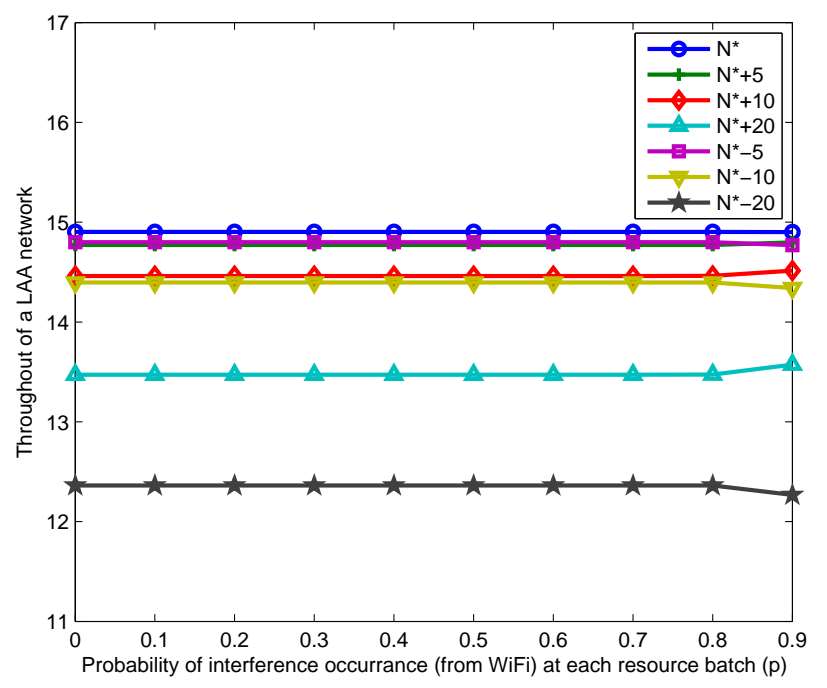

Fig. 3. Throughput of the LAA network under different number of LAA-UEs sharing a pool of 40 resource batches.

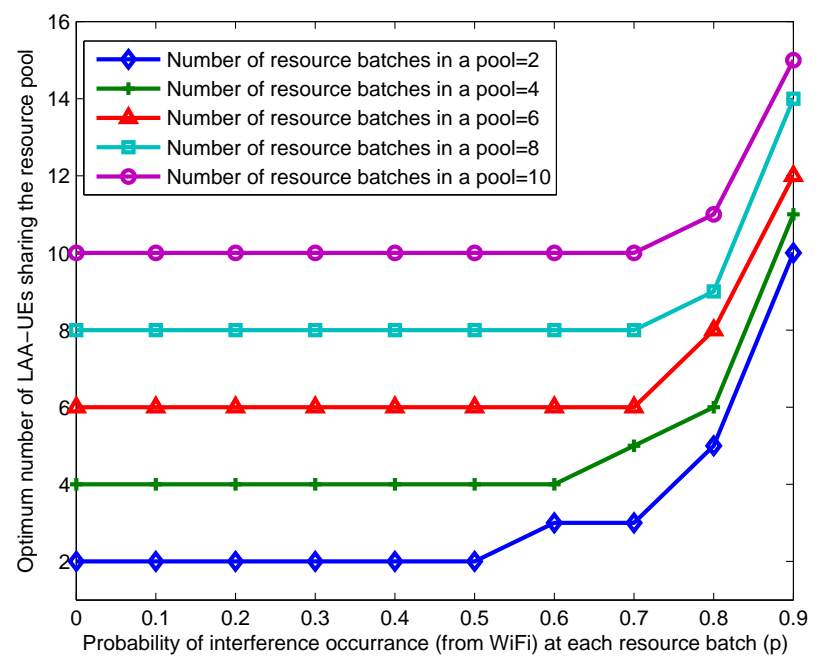

Fig. 4. The optimum numbers of LAA-UEs sharing different numbers of $M$ resource batches.

based radio resource access provides a better throughput as $p=1-\left(1-\frac{1}{M}\right)^{M-1} \approx 0.63$, which confirms Theorem 5 . By using this $p$ value, it shows the optimum switch between a scheduling based radio resource access and a random access. These results fully support our proposition.

\section{CONCLUSION}

In this paper, we analytically derive the optimum radio access scheme for LAA coexisting with WiFi networks as a switch between a conventional scheduling based radio resource access and a random access among a pool of resource batches. We analytically derive the optimum switching condition based on the activity of WiFi networks, and the optimum number of LAA-UEs sharing a pool of resource batches. The provided

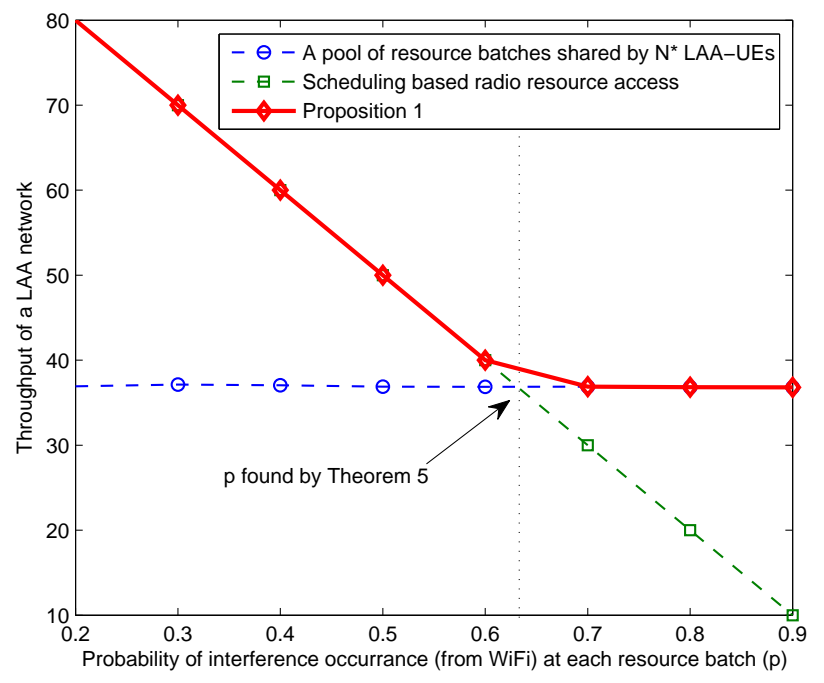

Fig. 5. The throughput of the LAA network using a random access, a scheduling based radio resource access, and our scheme in Proposition 1, where $M=100$

analytic as well as simulation results provides essential foundations for the urgent needs of the 3GPP LAA system design.

\section{REFERENCES}

[1] S.-Y. Lien, K.-C. Chen, and Y. Lin, "Toward ubiquitous massive accesses in 3GPP machine-to-machine communications," IEEE Commun. Mag., vol. 49, no. 4, pp. 66-74, Apr. 2011.

[2] S.-Y. Lien and K.-C. Chen, "Massive access management for QoS guarantees in 3GPP machine-to-machine communications," IEEE Commun. Lett., vol. 15, no. 3, pp. 311-313, Mar. 2011.

[3] Z. Ding, Z. Yang, P. Fan, and H. Poor, "On the performance of nonorthogonal multiple access in 5G systems with randomly deployed users," IEEE Signal Process. Lett., vol. 21, no. 12, pp. 1501-1505, Aug. 2014.

[4] J. Choi, "Minimum power multicast beamforming with superposition coding for multiresolution broadcast and application to NOMA systems," IEEE Trans. Commun., vol. 63, no. 3, pp. 791-800, Mar. 2015.

[5] E. Ahmend, A. Eltawil, and A. Sabharwal, "Rate gain region and design tradeoffs for full-duplex wireless communications," vol. 12, no. 7, pp. 3556-3565, Jul. 2013. 